Yervant Terzian, Daniel Weedman, Edward Khachikian, eds.

\title{
Radio Properties of Quasars and AGN
}

\author{
K. I. Kellermann
}

NRAO, 520 Edgemont Road, Charlottesville VA 22903

\begin{abstract}
We discuss the compact radio sources associated with quasars and AGN with particular emphasis on the implications of their structure and kinematics for relativistic beaming models.
\end{abstract}

\section{Introduction}

About ten to fifteen percent of optically selected quasars and AGN are radio loud defined by $\mathrm{P}_{6}>10^{25} \mathrm{~W} / \mathrm{Hz}$ or $\mathrm{R}>10$, where $\mathrm{P}_{6}$ is the radio flux density at $\lambda=6 \mathrm{~cm}$ and $\mathrm{R}$ is the ratio of radio to optical flux density measured at 6 $\mathrm{cm}$ and $4400 \mathrm{~A}$ respectively (Kellermann, et al. 1989; Miller et al. 1990). The radio loud quasars and AGN are conveniently divided into two categories, the lobe-dominated sources primarily associated with radio galaxies and the coredominated sources which are mostly identified with quasars and AGN. For both the lobe-dominated and core-dominated sources, the source of energy appears to lie in a compact central engine generally assumed to be due to accretion from a surrounding material onto a massive black hole. Radio emission from the extended lobes arises from optically thin steep spectrum synchrotron radiation which may extend up to hundreds of kiloparsecs from the parent galaxy. The core dominated sources, on the other hand, are dominated by very compact self-absorbed, often variable, synchrotron sources. High resolution radio observations give a unique opportunity to study in detail the processes occurring close to the central engine.

Much less is known about the nature of the radio quiet quasars and AGN. At least some of the low luminosity radio emission appears to be the result of intense star-forming activity, generally coincident with the visible optical disk and showing little or no detailed radio structure (e.g., Richards et al. 1998). However, some radio quiet quasars also have more extended radio lobes as well as compact radio components more characteristic of the activity of a central engine. It seems likely that the low luminosity radio emission observed from radio quiet objects is a combination of star-forming activity and ejection from a compact central engine.

At the time of the IAU Symposium No. 29 on Non Stable Phenomena in Galaxies which was held in Byurukan in 1966, it was realized that the rapid time variability observed in many quasars and AGN implied such small angular dimensions that corresponding physical conditions appeared to be unrealistic (Kellermann and Pauliny-Toth 1968). It is now appreciated that the observed radio emission from quasars and AGN can be understood in terms of the twin relativistic jet model described by Blandford and Konigl (1979) based on ideas 
first discussed by Shklovsky (1963) and later extended by Rees (1966) and Woltjer (1966). We note that only at radio wavelengths is it possible to see through the gas and dust close to the AGN where relativistic plasma is focused into a narrow beam or jet. Also, only at radio wavelengths, is it possible to directly observe the relativistic flow from the central engine.

There are three observational consequences of relativistic motion. First, of course, owing to the Doppler shift, the emission from the approaching beam is shifted toward shorter wavelengths, and the receding component toward longer wavelengths by the Doppler factor $\delta=1 / \gamma_{\mathrm{f}}(1-\beta \cos \theta)$. Second, as a result of aberration, the observed flux density from a relativistically moving source is boosted by a factor $\delta^{n+\alpha}$. Finally, because a relativistically moving source nearly catches up with its own radiation, the time scale is compressed for an observer located close to the direction of motion. The corresponding apparent transverse velocity, $\beta_{\mathrm{app}}=\beta_{\mathrm{p}} \sin \theta /\left(1-\beta_{\mathrm{p}} \cos \theta\right)$, and can exceed the speed of light when $2 \beta_{\mathrm{p}} /\left(1+\beta_{\mathrm{p}}^{2}\right)>\cos \theta$. In these expressions, $\gamma=\left(1-\beta^{2}\right)^{-1 / 2}$ is the usual Lorentz factor, $\beta=\mathrm{v} / \mathrm{c} ; \theta$ is the angle between the motion and the line of sight; the subscripts $f$ and $p$ refer respectively to the relativistic flow velocity of the radiating source and the velocity of the moving pattern; and $n=2$ or 3 depending on whether the motion is in the form of a continuous jet or discrete components.

The effect of differential Doppler boosting between the approaching and receding sources was the basis of the original unified models which attempt to describe the different appearance of lobe-dominated and core-dominated sources as the result of the different orientation of intrinsically similar sources containing both extended unbeamed radio lobes as well as compact relativistic jets (Orr \& Browne 1982). Later Antonucci and Miller (1985), and Barthel (1989) included the effect of observation of the nucleus by a dusty torus surrounding the central engine to extend the unified picture to optical wavelengths.

In the simplest relativistic beaming models, $\gamma_{\mathrm{f}}=\gamma_{\mathrm{p}}$ and there are simple relations between the observed component velocities and the degree of Doppler boosting which allow quantitative tests of the beaming models and the intrinsic velocity and orientation to be uniquely determined from observation of the apparent component motion and flux density ratio of the approaching and receding components. In this paper we briefly describe the high resolution observations of quasars and AGN and their implication to the nature of the compact central engine and the formation and propagation of relativistic jets. We assume throughout $\mathrm{H}_{0}=65 / \mathrm{km} / \mathrm{sec} / \mathrm{Mpc}$ and $\mathrm{qo}=1 / 2$. Other recent relevant reviews can be found in Cohen \& Kellermann (1995), Zensus (1997), Zensus et al. (1998), and Urry and Padovannini (1995).

\section{Morphology}

High resolution radio observations using the Very Long Baseline Array (VLBA), the European Very Long Baseline Network (EVN), and the Southern Hemisphere (SHEVE) give images of the radio emission near the central engine with an angular resolution exceeding 0.001 arcseconds ( 1 milliarcsec), or orders of magnitude better than images made with any other instrument operating in any wavelength band, on the ground or in space. The corresponding linear scale is only a few 
tens of parsecs even at cosmological distances $(\mathrm{z} \sim 1)$ and only a few tenths of a parsec for nearby $(\mathrm{z} \sim 0.01)$ galaxies. For the more powerful quasars and AGN with $\mathrm{P}_{6}>10^{25} \mathrm{~W} / \mathrm{Hz}$ the structure is invariably one-sided, with the parsec scale jet lying on the same side as the much larger kiloparsec scale jets which feed the extended radio lobes. It is assumed that the kiloparsec scale jets are intrinsically two-sided as suggested by the symmetric appearance of the radio lobes. Thus, if the one-sided appearance of the kiloparsec jet is due to differential Doppler boosting, the kiloparsec scale jets must also be relativistic, and must be focused on scales of a few parsecs or less, yet remain collimated out to hundreds of kiloparsecs.

Earlier VLBI observations suggested a radio morphology which consisted of a compact core with multiple secondary components lying along a jet-like structure. The new VLBA (e.g., Kellermann et al. 1998a) and EVN observations, which have greater sensitivity to low surface brightness structure, often show a complex continuous jet with condensations possibly the result of shock fronts. The compact core is self absorbed, sometimes even at short centimeter or millimeter wavelengths. The jets are sometimes very narrow and appear unresolved in the transverse direction with linear dimensions of the order of a few parsecs or less. Often, the jets have multiple bends or twists, sometimes up to 90 degrees or more, especially close to the core. Some jets show an oscillating appearance characteristic of a rotating nozzle or the effect of Kelvin-Helmholtz instabilities. These one-sided compact structures typically have a flat radio spectrum or one with multiple maxima and minima which are thought to be the superposition of multiple components each with a different self absorption cutoff frequency.

Some AGN as well as a few quasars show symmetric structure up to hundreds of parsecs in extent. These compact symmetric objects (CSOs) frequently contain a very compact central component which we identify with the center of activity (Readhead et al. 1996). CSOs appear to be related to the class of source with $\mathrm{GHz}$ peaked spectra (GPS) with a spectral peak near one GHz. It seems likely that the spectral peak is due to synchrotron self absorption, but we can not rule out the presence of free-free absorption by an intervening ionized medium. Possible interpretations of CSOs and GPS sources which have been discussed include a) gravitational "minilensing" from black holes of the order of $10^{6} \mathrm{M}_{\odot}$ located in galactic halos (Lacey \& Ostriker 1985); b) young classical doubles (e.g., Phillips and Mutel 1980); c) "frustrated" or confined doubles (O'Dea et al. 1991); d) reborn doubles (Baum et al. 1990); e) smothered doubles (Baum et al 1990); or f) intrinsically confined doubles (Gopal-Krishna \& Wiita (1991).

Often, the low luminosity AGN show a bilateral or two-sided jet symmetry at short wavelengths but at longer wavelengths, the structure may appear asymmetric due to free-free absorption in the nuclear region, apparently by ionized circumnuclear gas contained in a torus or other structure oriented perpendicular to the jet (Levinson, et al. 1995; Walker, et al.1998, Kellermann et al. 1998a). In these sources, the jets appear to propagate through a rich and complex medium, possibly including the broad-line region and containing both molecular (e.g., Claussen et al. 1998) and atomic gas (Vermeulen et al. 1999). The AGN found in NGC 5128 (Jones, et al. 1996), NGC 1052 (Kellermann, et 
al. 1998b), NGC 1275 (Dhawan, et al. 1998), and Cygnus A (Krichbaum, et al. 1998) all show signs of asymmetric absorption from an intervening medium.

High resolution polarimetry to determine the distribution of linear polarization gives further insight into the strength and orientation of the magnetic field in quasars and AGN as well as other information about jet physics (e.g., Wardle et al. 1994). In general the orientation of the magnetic field is aligned with the jet direction for quasars but tends to be orthogonal to the jet in weak lined sources such as those associated with BL Lac objects (Cawthorne et al. 1993).

Until recently, it has been unclear whether relativistic jets are composed of a "normal" electron-proton plasma or electron-positron pairs. Wardle et al. (1998), have reported the detection of circular polarization in several quasars and AGN which they argue is evidence that the relativistic jets must be composed primarily of electrons and positrons. Future observations of circular polarization combined with measurements of Faraday rotation of linear polarization will give new insight on the energy and mass content of relativistic jets and help to understand how they are created.

\section{Kinematics}

The observed peak radio brightness temperatures of quasars and AGN are typically in the range of $10^{11-12} \mathrm{~K}$. The maximum brightness temperature for a static incoherent synchrotron source is limited by inverse Compton cooling to about $3 \times 10^{11} \mathrm{~K}$ (Kellermann \& Pauliny-Toth 1969) and somewhat less if there is equilibrium between the relativistic particles and the magnetic field (Readhead 1994). But the observed values can be larger if there is a coherent mechanism involved, if the source is not stationary (Slish 1992), or if Doppler boosting is significant. Comparison of inverse Compton scattered $x$-ray emission with observed peak brightness temperatures can be used to estimate the amount of Doppler boosting. Ghisellini et al. 1993 find good agreement with inverse Compton Doppler factors and observed superluminal velocities, but Guerra et al. (1997) find better agreement with equipartition Doppler factors and observed motions. Recently, Bower \& Backer (1998) have used space VLBI observations to directly measure a peak brightness temperature of about $3 \times 10^{12} \mathrm{~K}$ in the quasar NRAO 530 significantly in excess of inverse Compton or equilibrium values expected from a static source.

When examined over a period of time, the flux density of most quasars and AGN show variability on time scales ranging from a few hours to a few years. The most rapid so-called "intraday" variables (Heeschen et al. 1987, Wagner \& Witzel 1996, Wagner 1998) present a challenge to standard models. If the variations are intrinsic as suggested by the similarity of observed variations over a wide range of wavelength (Wagner et al. 1996), light travel time arguments suggest extremely small dimensions corresponding to brightness temperatures as great as $10^{21} \mathrm{~K}$. Assuming synchrotron radiation, such large brightness temperatures require unrealistic large values of the Doppler factor up to $10^{3}$ (Kedziora-Chudczer et al 1998), non stationary conditions in the relativistic plasma (Slish 1992), or coherent emission mechanisms. It has been argued that the observed rapid time variations may not be intrinsic but may be due to 
refractive scintillations in the interstellar medium. However, in order that such rapid scintillations occur, the angular size is still limited to about 5 as for the most rapid sources and the corresponding brightness temperatures are still an uncomfortably high value of $10^{14} \mathrm{~K}$ (Cordes 1998).

Corresponding to the observed changes in total flux density, individual features appear to be ejected from the central engine with apparent linear velocities mostly in the range $0<\mathrm{v} / \mathrm{c}<10$ with a median value about $3 \mathrm{c}$. Radio sources associated with strong gamma-ray sources have somewhat faster moving components suggesting either a larger intrinsic velocity or motion more closely aligned to the line of sight. In every case with a well determined velocity, the motion is outward along the jet. It is perhaps surprising that there is no direct evidence from the radio data of the postulated infall into a massive black hole; only outflows are observed.

Compact radio sources at the center of symmetric double lobed quasars typically have slower velocities than the core-dominated asymmetric sources (e.g., Hough 1996) as expected from unified models that place the lobe dominated sources near the plane of the sky. Motions have been observed in only a few FR II radio galaxies. In Cygnus $\mathrm{A}$ the motion is surprisingly subluminal (Krichbaum et al. 1998), while in 3C 111 and 3C 390.3 it is superluminal (Kellermann et al. 1998 b) with observed velocities comparable to typical core dominated quasars.

The low luminosity components in AGN appear to have significantly slower moving components. For a given luminosity, there appears to be no difference in component velocities found in BL Lacs, AGN, and quasars. This is perhaps surprising, since the relativistic beaming models consider BL Lacs, AGN, and quasars to be increasingly aligned along the line of sight so that the observed kinematics should differ among these populations. Moreover, in the two-sided low luminosity jets, the observed transverse velocities are often much less than the speed of light. AGN in the galaxies NGC 1052, M87, Cygnus A, and NGC 5128 show velocities in the range $0.1 \mathrm{c}$ to $0.5 \mathrm{c}$ (Kellermann, et al. $1998 \mathrm{~b}$; Tingay et al. 1998; Krichbaum, et al. 1998).

Relativistic beaming models all require a common intrinsic velocity very close to the speed of light. From Section 1, we see that according to the twin-jet model, the apparent transverse velocity cannot be less than the speed of light for any orientation. It is therefore difficult to understand how these two-sided compact sources or the asymmetric cores of the symmetric lobe-dominated FR II galaxies fit into the simple unified beaming models which consider the low radio luminosity AGN and the lobe dominated quasars and FR II radio galaxies to differ from the high luminosity quasars only by orientation. Rather, it would appear that there are intrinsic differences as well.

Individual sources often show apparent accelerations along the jet, e.g., M87, NGC 1275, Cygnus A, 3C 345, but it is not clear if these represent real changes in velocity, or are just the effect of motion along a curved trajectory with a changing inclination to the line of sight (Dhawan, et al. 1998; Krichbaum, et al. 1998; Unwin et al. 1997).

In a randomly oriented sample, most sources should lie close to the plane of the sky so the observed transverse velocity of the jet components should be close to $c$ (or $2 c$ if measured between oppositely moving jets. On the other hand, in a flux density limited sample, differential Doppler boosting results in a 
bias toward sources moving close to the line of sight. In this case, the observed distribution of apparent velocities depends in detail on the intrinsic value of the Lorentz factor, $\gamma$, the spectral index, $\alpha$, and the slope of the radio source count. Vermeulen (1995) has shown that for a wide range of parameters the observed distribution of apparent velocity should be closely peaked near a value of $\gamma$ c. The observed distribution of apparent linear velocity is not consistent with any simple ballistic model and appears to require either a spread in intrinsic velocity or a difference between flow and pattern velocities (Vermeulen 1995, Kellermann et al. 1998b). Lister \& Marscher (1997) discuss the observed statistics of superluminal velocities in flux limited samples of radio sources.

Acknowledgments. Some of the results described here are based on unpublished work done in collaboration with R. Vermeulen, J. A. Zensus, and M. Cohen. The National Radio Astronomy Observatory is a facility of the National Science Foundation, operated under a cooperative agreement by Associated Universities, Inc.

\section{References}

Antonucci \& Miller 1985. ApJ, 297, 621.

Barthel, P. 1989. ApJ, 336, 606.

Baum, S.A., 1990, A\&A, 232, 19.

Blandford, R.D. \& Konigl, A. 1979. ApJ, 232, 34.

Bower, G.C. \& Backer, D.C. 1998, ApJ, 507, L117.

Cawthorne, T.V. et al. 1993. ApJ, 416, 519.

Cohen, M.H. \& Kellermann, K.I. 1995. Proc.of the Nat. Academy of Sciences, 92,11339 .

Claussen, et al. 1998, ApJ, 500, L129.

Cordes, J. 1998. in Radio Emission from Compact Galactic and Extragalactic Radio Sources, eds. A. Zensus et al., ASP., San Francisco, p. 329.

Dhawan, V. et al. 1998, ApJ, 498, L111.

Ghisellini, G. et al. 1993, ApJ407, 65.

Gopal-Krishna \& Wiita, P. J. 1991, ApJ, 373, 325.

Guerra, E. et al. 1997. ApJ, 491, 483.

Hough, D. et al. 1996. ApJ, 459, 64.

Heeschen, D.S., et al. 1987. AJ, 94, 1493.

Jones, D. et al. 1996, ApJ, 466, L63.

Kedziora-Chudczer, L.L. et al. 1998. in Radio Emission from Compact Galactic and Extragalactic Radio Sources, eds. A. Zensus et al., ASP., San Francisco, p. 267

Kellermann, K.I. \& Pauliny-Toth, I.I.K. 1968, in Non-Stable Phenomena in Galaxies, Academy of Sciences of Armenian SSR, Yerevan, p. 245.

Kellermann, K.I., et al. 1989, AJ, 98, 1185.

Kellermann, K.I. et al. 1998a. AJ, 115, 1295. 
Kellermann, K.I., et al. 1998b, in Proc. of EVN/JIVE Symp. No. 4, New Astron. Rev., in press.

Krichbaum, T.P., et al. 1998, A\&A, 329, 873.

Lacey, C. G. \& Ostriker, J.P. 1985, ApJ, 299, 649.

Levinson, A., et al. 1995, ApJ, 448, 589.

Lister, M.L. \& Marscher, A.P. 1997. ApJ, 476, 572.

Miller, L. et al. 1990. MNRAS, 244, 207.

O'Dea, C.P. et al.1991, ApJ, 380, 66.

Orr, M.J.W. \& Browne, I.W.A. 1982. MNRAS, 200, 1067.

Phillips, R.B. \& Mutel, R.L. 1980, ApJ, 236, 89.

Readhead, A.C.S. 1994. ApJ, 426, 51.

Readhead, A.C.S. et al. 1996. ApJ, 460, 612.

Rees, M. 1966. Nature, 211, 468.

Richards, E.R., et al., 1998. AJ, 116, 1039.

Shklovsky, J. 1963, Sov.-Astron., 7, 748.

Slish, V.I. 1992. ApJ, 391, 453.

Tingay, S. et al. 1998, Aj, 115, 960.

Unwin et al. 1997. ApJ, 480, 596.

Urry, M. \& Padovannini, 1995. PASP, 107, 803.

Vermeulen, R.C. 1995. Proceedings of the National Academy of Sciences, 92, 11385

Vermeulen, R.C. 1999, in preparation.

Wagner, S.J. et al. 1996. AJ, 111, 2187.

Wagner, S.J. 1998, in Radio Emission from Compact Galactic and Extragalactic Radio Sources, eds. A. Zensus et al., ASP., San Francisco, p. 257

Wagner, S.J. \& Witzel, A. 1995, ARA\&A, 33, 163.

Walker, R.C. et al. 1998, in Radio Emission from Compact Galactic and Extragalactic Radio Sources, eds. A. Zensus et al., AS., San Francisco, p. 133

Wardle, J.F.C. et al. 1994. ApJ, 437, 122.

Wardle, J. 1998. Nature, 395, 457.

Wotjer, L. 1966, ApJ, 146, 597.

Zensus, J.A. 1997. ARA\&A. 33, 607.

Zensus et al. 1998, Proceedings of IAU Colloquium No. 164, Radio Emission from Compact Galactic and Extragalactic Radio Sources, Astron. Soc. Pac., San Francisco. 